\title{
Multiplying balls in the space of continuous functions on $[0,1]$
}

\author{
by \\ Marek Balcerzak, Artur Wachowicz and \\ WŁADYSŁAW WILCZYŃSKI (Łódź)
}

\begin{abstract}
Let $C$ denote the Banach space of real-valued continuous functions on $[0,1]$. Let $\Phi: C \times C \rightarrow C$. If $\Phi \in\{+, \min , \max \}$ then $\Phi$ is an open mapping but the multiplication $\Phi=$. is not open. For an open ball $B(f, r)$ in $C$ let $B^{2}(f, r)=B(f, r)$. $B(f, r)$. Then $f^{2} \in \operatorname{Int} B^{2}(f, r)$ for all $r>0$ if and only if either $f \geq 0$ on $[0,1]$ or $f \leq 0$ on $[0,1]$. Another result states that $\operatorname{Int}\left(B_{1} \cdot B_{2}\right) \neq \emptyset$ for any two balls $B_{1}$ and $B_{2}$ in $C$. We also prove that if $\Phi \in\{+, \cdot, \min , \max \}$, then the set $\Phi^{-1}(E)$ is residual whenever $E$ is residual in $C$.
\end{abstract}

1. Openness of some operations. Let $B(x, r)$ denote the (open) ball with centre $f$ and radius $r$ in a metric space. By Int we denote the interior. Let $C=C[0,1]$ stand for the Banach algebra of real-valued continuous functions on $[0,1]$ with the norm $\|f\|=\sup _{x \in[0,1]}|f(x)|, f \in C$. Let $\Phi: C \times C \rightarrow C$ be one of the four operations: addition (+), multiplication $(\cdot)$, minimum (min), and maximum (max). For two balls $B_{1}=B(F, r)$ and $B_{2}=B(G, r)$ in $C$, it is natural to ask what is the image $\Phi\left(B_{1} \times B_{2}\right)$. Now, let us simplify some notation. If $\Phi \in\{+, \cdot, \min , \max \}$ and $E_{1}, E_{2} \subset C$, we denote the respective images by $E_{1}+E_{2}, E_{1} \cdot E_{2}, \min \left(E_{1}, E_{2}\right), \max \left(E_{1}, E_{2}\right)$. The answer to our question for addition, minimum and maximum is simple. The case of multiplication is nontrivial and more interesting.

First we study the behaviour of the sum of two balls in an arbitrary normed space:

Proposition 1. For an arbitrary normed space $X$ we have

$$
B\left(x_{1}, r_{1}\right)+B\left(x_{2}, r_{2}\right)=B\left(x_{1}+x_{2}, r_{1}+r_{2}\right)
$$

where $x_{1}, x_{2} \in X$ and $r_{1}, r_{2}>0$.

Proof. The inclusion " $\subset$ " is clear. For the reverse inclusion, consider $z \in$ $B\left(x_{1}+x_{2}, r_{1}+r_{2}\right)$ and put

2000 Mathematics Subject Classification: 46J10, 46B25, 26A15, 54E52.

Key words and phrases: Banach algebra, multiplication, continuous function, polygonal function, residual set. 


$$
z_{i}=x_{i}+\frac{r_{i}}{r_{1}+r_{2}}\left(z-\left(x_{1}+x_{2}\right)\right) \quad \text { for } i=1,2 .
$$

Then $z_{i} \in B\left(x_{i}, r_{i}\right)$ for $i=1,2$ and $z=z_{1}+z_{2}$.

Proposition 2. For every $F, G \in C$ and $r>0$ we have

$$
\begin{gathered}
\min (B(F, r), B(G, r))=B(\min (F, G), r), \\
\max (B(F, r), B(G, r))=B(\max (F, G), r) .
\end{gathered}
$$

Proof. For instance, let us show the first equality. To demonstrate " $\subset$ ", let $h=\min (f, g)$ where $f \in B(F, r)$ and $g \in B(G, r)$. Hence

$$
F(x)-r<f(x)<F(x)+r \quad \text { and } \quad G(x)-r<g(x)<G(x)+r
$$

for all $x \in[0,1]$. Consequently, for all $x \in[0,1]$ we have

$$
\min (F, G)(x)-r<h(x)<\min (F, G)(x)+r .
$$

Since the above functions are continuous on $[0,1]$, we have

$$
\sup _{x \in[0,1]}|h(x)-\min (F, G)(x)|<r
$$

and so, $h \in B(\min (F, G), r)$ as desired.

For the reverse inclusion, let $h \in B(\min (F, G), r)$. For each $x \in[0,1]$ put $\alpha(x)=h(x)-\min (F, G)(x), \quad f(x)=F(x)+\alpha(x), \quad g(x)=G(x)+\alpha(x)$. Then $\|\alpha\|<r$ and consequently, $f \in B(F, r)$ and $g \in B(G, r)$. Since also $h=\min (f, g)$, we have $h \in \min (B(F, r), B(G, r))$.

Remark. Consider $F(x)=x, G(x)=1-x$ for $x \in[0,1]$ and $r_{1}=$ $1 / 4, r_{2}=1 / 2$. It is easy to check that neither $\min \left(B\left(F, r_{1}\right), B\left(G, r_{2}\right)\right)$ nor $\max \left(B\left(F, r_{1}\right), B\left(G, r_{2}\right)\right)$ is a ball. Nevertheless, from Propositions 1 and 2 it follows that every mapping $\Phi \in\{+, \min , \max \}$ is open. We will see that for multiplication, this is not true.

For $f \in C$ and $r>0$ we write $B^{2}(f, r)=B(f, r) \cdot B(f, r)$. In January 2004, D. H. Fremlin (oral communication) observed that for $f(x)=x-1 / 2$, $x \in[0,1]$, one has

$$
f^{2} \in B^{2}(f, 1 / 2) \backslash \operatorname{Int} B^{2}(f, 1 / 2) .
$$

Hence multiplication is not an open mapping from $C \times C$ into $C$.

We have the following characterization.

TheOREM 3. Let $f \in C$. Then $f^{2} \in \operatorname{Int} B^{2}(f, r)$ for all $r>0$ if and only if either $f \geq 0$ on $[0,1]$ or $f \leq 0$ on $[0,1]$.

Proof. " $\Rightarrow$ " (This part is inspired by Fremlin's example.) Suppose to the contrary that there are $a, b \in[0,1]$ such that $f(a)<0$ and $f(b)>0$. Define $r=\min \{|f(a)|,|f(b)|\}$. Let $g \in B(f, r)$. Thus $g(a)<0$ and $g(b)>0$, and by the Darboux property, $g$ has a zero between $a$ and $b$. Hence every function 
in $B^{2}(f, r)$ has a zero in $[0,1]$. By assumption, $f^{2} \in \operatorname{Int} B^{2}(f, r)$. Hence $f^{2}+\varepsilon \in B^{2}(f, r)$ for a sufficiently small $\varepsilon>0$. But $f^{2}+\varepsilon>0$ on $[0,1]$, which contradicts our previous observation.

" $\Leftarrow$ " We may assume that $f \geq 0$ on $[0,1]$ since, if $f \leq 0$ on $[0,1]$, we use $-f$ and notice that $B^{2}(f, r)=B^{2}(-f, r)$. Fix $r>0$ and put $\varepsilon=r^{2} / 9$. We will show that $B\left(f^{2}, \varepsilon\right) \subset B^{2}(f, r)$. Let $g \in B\left(f^{2}, \varepsilon\right)$. Then

$$
f^{2}(x)-\varepsilon<g(x)<f^{2}(x)+\varepsilon
$$

for each $x \in[0,1]$. We have $g=g_{1} g_{2}$ on $[0,1]$ where $g_{1}=\sqrt{|g|}, g_{2}=$ $\sqrt{|g|} \operatorname{sgn} g$. Obviously $g_{1}, g_{2} \in C$. From (1) it follows that $|g(x)|<f^{2}(x)+\varepsilon$ for each $x \in[0,1]$ and consequently,

$$
\sqrt{|g(x)|}<\sqrt{f^{2}(x)+\varepsilon} \leq f(x)+\sqrt{\varepsilon} .
$$

On the other hand, using again (1), for all $x \in[0,1]$ we have $|g(x)|+$ $2 \sqrt{|g(x)| \varepsilon}+\varepsilon \geq g(x)+\varepsilon>f^{2}(x)$. Hence $(\sqrt{|g(x)|}+\sqrt{\varepsilon})^{2}>f^{2}(x)$ and thus $\sqrt{|g(x)|}>f(x)-\sqrt{\varepsilon}$. This together with (2) shows that $g_{1} \in B(f, \sqrt{\varepsilon}) \subset$ $B(f, r)$.

To show that $g_{2} \in B(f, r)$ it is enough to consider the case when $g(x)<0$ for a fixed $x \in[0,1]$. Then by (1) we have $f^{2}(x)-\varepsilon<g(x)<0$. So $f(x)<\sqrt{\varepsilon}$ and $2 f(x)<2 \sqrt{\varepsilon}=2 r / 3=r-\sqrt{\varepsilon}$. Hence $-f(x)-\sqrt{\varepsilon}>f(x)-r$ and from (2) we get

$$
-\sqrt{|g(x)|}>-f(x)-\sqrt{\varepsilon}>f(x)-r .
$$

Also obviously, $-\sqrt{|g(x)|}<f(x)+r$. Thus $g_{2} \in B(f, r)$.

Corollary 4. The set $\left\{f \in C:(\forall r>0) f^{2} \in \operatorname{Int} B^{2}(f, r)\right\}$ is closed.

2. Weak openness of multiplication. Although multiplication from $C \times C$ into $C$ is not an open mapping, it always transforms each open nonempty set onto a set with nonempty interior. Let us call this property weak openness. Namely, we have the following general result:

Theorem 5. $\operatorname{Int}(B(F, R) \cdot B(G, R)) \neq \emptyset$ for all $F, G \in C$ and $R>0$.

Of course, it suffices to assume that the centres $F, G$ come from a fixed dense set in $C$. It is convenient to take a dense set of all polygonal functions in $C$. More precisely, we consider the set $P$ of all polygonal functions $f$ in $C$ such that $f(0) \neq 0, f(1) \neq 0$ and $f^{-1}(\{0\})$ does not contain interior points. It is easy to check that $P$ is also dense in $C$.

Lemma 6. Let $F, G \in P$ and $r>0$. There are a partition $0=x_{0}<x_{1}<$ $\cdots<x_{2 n}=1$ of $[0,1]$ and functions $f, g \in P$ such that:

- $\|f-F\|<r$ and $\|g-G\|<r$;

- $f$ and $g$ are affine on every interval $I_{i}=\left[x_{i-1}, x_{i}\right]$ for $i=1, \ldots, 2 n$; 
- $\left.f\right|_{I_{k}}=$ const $=a_{k} \neq 0$ for each odd $k \in\{1, \ldots, 2 n\}$, $\left.g\right|_{I_{k}}=$ const $=a_{k} \neq 0$ for each even $k \in\{1, \ldots, 2 n\}$.

Proof. By the uniform continuity of $F$ and $G$ we choose $\delta>0$ such that $\left|F(x)-F\left(x^{\prime}\right)\right|<r$ and $\left|G(x)-G\left(x^{\prime}\right)\right|<r$ for any $x, x^{\prime} \in[0,1]$ with $\left|x-x^{\prime}\right|<\delta$. Since $F, G \in P$, we can pick a partition $0=x_{0}<x_{1}<\cdots<$ $x_{2 n}=1$ with diameter $<\delta / 2$ such that $F\left(x_{i}\right) \neq 0$ and $G\left(x_{i}\right) \neq 0$ for $i=1, \ldots, 2 n$. Put $f(1)=F(1), g(0)=G(0)$, and $\left.f\right|_{I_{k}}=F\left(x_{k-1}\right)$ if $k \in$ $\{1, \ldots, 2 n\}$ is odd, $\left.g\right|_{I_{k}}=G\left(x_{k-1}\right)$ if $k \in\{1, \ldots, 2 n\}$ is even. Then we extend $f$ and $g$ to be continuous on $[0,1]$ and affine on every interval $I_{i}$. Finally, we define

$$
a_{k}= \begin{cases}F\left(x_{k-1}\right) & \text { if } k \in\{1, \ldots, 2 n\} \text { is odd } \\ G\left(x_{k-1}\right) & \text { if } k \in\{1, \ldots, 2 n\} \text { is even. }\end{cases}
$$

Lemma 7. Let $f, g \in P$ and $x_{i}(i=0, \ldots, 2 n)$ be as in Lemma 6. Define

$$
\begin{aligned}
& \mu=\min \left\{\left|f\left(x_{i}\right) g\left(x_{i}\right)\right|: i=0, \ldots, 2 n\right\}, \\
& \gamma=\max \left\{\left|f\left(x_{i}\right) g\left(x_{i}\right)\right|: i=0, \ldots, 2 n\right\} .
\end{aligned}
$$

Then for all $\varepsilon \in(0, \mu / 2], \varphi \in B(f g, \varepsilon)$ and $i \in\{0, \ldots, 2 n\}$ we have

$$
\mu / 2<\left|\varphi\left(x_{i}\right)\right|<\gamma+\mu / 2 .
$$

Proof. We have

$$
\begin{aligned}
& \left|\varphi\left(x_{i}\right)\right| \geq\left|f\left(x_{i}\right) g\left(x_{i}\right)\right|-\|\varphi-f g\|>\mu-\varepsilon \geq \mu / 2, \\
& \left|\varphi\left(x_{i}\right)\right| \leq\left|f\left(x_{i}\right) g\left(x_{i}\right)\right|+\|\varphi-f g\|<\gamma+\varepsilon \leq \gamma+\mu / 2 .
\end{aligned}
$$

From now on, for $h \in C$ we write $\|h\|_{i}=\sup _{x \in I_{i}}|h(x)|, i=1, \ldots, 2 n$.

Lemma 8. Keeping all notations from Lemmas 6 and 7 , one can find numbers $\beta_{i}>0(i=1, \ldots, 2 n)$ such that for each $\varepsilon \in(0, \mu / 2]$ and for every $\varphi \in B(f g, \varepsilon)$ there are functions $\zeta, \psi \in C$ with $\varphi=\zeta \psi$ and $\|f-\zeta\|_{i}<\beta_{i} \varepsilon$, $\|g-\psi\|_{i}<\beta_{i} \varepsilon$ for $i=1, \ldots, 2 n$.

Proof. We will use induction with respect to $i \in\{1, \ldots, 2 n\}$. Step by step, we will define $\beta_{i}$ and (for given $\varepsilon$ and $\varphi$ ) we will define $\left.\zeta\right|_{I_{i}}$ and $\left.\psi\right|_{I_{i}}$ in such a way that $\left.\zeta\right|_{I_{i}}=$ const $=d_{i}$ if $i$ is odd, and $\left.\psi\right|_{I_{i}}=$ const $=d_{i}$ if $i$ is even.

Let $d_{1}=a_{1}$. Define $\zeta$ and $\psi$ on $I_{1}$ by $\zeta=$ const $=d_{1}$ and $\psi=\left(1 / d_{1}\right) \varphi$. Thus $\zeta \psi=\varphi$ on $I_{1}$. Additionally $\|f-\zeta\|_{1}=0$ and

$$
\begin{aligned}
\|g-\psi\|_{1} & =\left\|g-\frac{1}{d_{1}} \varphi\right\|_{1}=\frac{1}{\left|d_{1}\right|}\left\|d_{1} g-\varphi\right\|_{1} \\
& =\frac{1}{\left|d_{1}\right|}\|f g-\varphi\|_{1} \leq \frac{1}{\left|d_{1}\right|}\|f g-\varphi\|<\frac{1}{\left|d_{1}\right|} \varepsilon .
\end{aligned}
$$

Put $\beta_{1}=1 /\left|d_{1}\right|=1 /\left|a_{1}\right|$. 
We define $\zeta$ and $\psi$ on $I_{2}$. We want $\psi$ to be constant on $I_{2}$. Since $\psi$ should be continuous at $x_{1}$, we put

$$
\psi(x)=\psi\left(x_{1}\right)=\frac{1}{d_{1}} \varphi\left(x_{1}\right) \quad \text { for } x \in I_{2} .
$$

Thus $d_{2}=\left(1 / d_{1}\right) \varphi\left(x_{1}\right)=\left(1 / a_{1}\right) \varphi\left(x_{1}\right)$. We will estimate $\left|d_{2}\right|$. Pick a positive integer $N$ such that $1 / N \leq \mu / 2$ and $N \geq \gamma+\mu / 2$. By Lemma 7 we have

$$
\frac{1}{N\left|a_{1}\right|} \leq\left|d_{2}\right| \leq \frac{N}{\left|a_{1}\right|}
$$

To obtain $\varphi=\zeta \psi$ on $I_{2}$, we must put $\zeta=\left(1 / d_{2}\right) \varphi$ on $I_{2}$. We thus have

$$
\|g-\psi\|_{2}=\left|a_{2}-d_{2}\right|=\left|g\left(x_{1}\right)-\psi\left(x_{1}\right)\right| \leq\|g-\psi\|_{1}<\beta_{1} \varepsilon
$$

and

$$
\begin{aligned}
\|f-\zeta\|_{2} & =\frac{1}{\left|d_{2}\right|}\left\|d_{2} f-d_{2} \zeta\right\|_{2}=\frac{1}{\left|d_{2}\right|}\left\|d_{2} f-\varphi\right\|_{2} \\
& \leq \frac{1}{\left|d_{2}\right|}\left(\left|d_{2}-a_{2}\right|\|f\|_{2}+\left\|a_{2} f-\varphi\right\|_{2}\right) \\
& \leq \frac{1}{\left|d_{2}\right|}\left(\left|d_{2}-a_{2}\right|\|f\|_{2}+\|g f-\varphi\|\right) \\
& <\frac{1}{\left|d_{2}\right|}\left(\beta_{1} \varepsilon\|f\|+\varepsilon\right)=\frac{\varepsilon}{\left|d_{2}\right|}\left(\beta_{1}\|f\|+1\right) .
\end{aligned}
$$

Hence by (3) we obtain

$$
\|f-\zeta\|_{2} \leq \varepsilon N\left|a_{1}\right|\left(\beta_{1}\|f\|+1\right) .
$$

It is enough to put $\beta_{2}=\max \left\{\beta_{1}, N\left|a_{1}\right|\left(\beta_{1}\|f\|+1\right)\right\}$.

Now, we define $\zeta$ and $\psi$ on $I_{3}$. We want $\zeta$ to be constant on $I_{3}$. Since $\zeta$ should be continuous at $x_{2}$, we put $\zeta(x)=\zeta\left(x_{2}\right)=\left(1 / d_{2}\right) \varphi\left(x_{2}\right)$ for $x \in I_{3}$. Thus $d_{3}=\left(1 / d_{2}\right) \varphi\left(x_{2}\right)$. By $(3)$ and Lemma 7 we have

$$
\left|a_{1}\right| / N^{2} \leq\left|d_{3}\right| \leq N^{2}\left|a_{1}\right| .
$$

To obtain $\varphi=\zeta \psi$ on $I_{3}$, we must put $\psi=\left(1 / d_{3}\right) \varphi$ on $I_{3}$. We thus have

$$
\|f-\zeta\|_{3}=\left|a_{3}-d_{3}\right|=\left|f\left(x_{2}\right)-\zeta\left(x_{2}\right)\right| \leq\|f-\zeta\|_{2}<\beta_{2} \varepsilon
$$

and

$$
\begin{aligned}
\|g-\psi\|_{3} & =\frac{1}{\left|d_{3}\right|}\left\|d_{3} g-d_{3} \psi\right\|_{3}=\frac{1}{\left|d_{3}\right|}\left\|d_{3} g-\varphi\right\|_{3} \\
& \leq \frac{1}{\left|d_{3}\right|}\left(\left|d_{3}-a_{3}\right|\|g\|_{3}+\left\|a_{3} g-\varphi\right\|_{3}\right) \\
& \leq \frac{1}{\left|d_{3}\right|}\left(\left|d_{3}-a_{3}\right|\|g\|+\|f g-\varphi\|\right) \\
& <\frac{1}{\left|d_{3}\right|}\left(\beta_{2} \varepsilon\|g\|+\varepsilon\right)=\frac{\varepsilon}{\left|d_{3}\right|}\left(\beta_{2}\|g\|+1\right) .
\end{aligned}
$$


Hence by (4) we obtain

$$
\|g-\psi\|_{3} \leq \frac{\varepsilon N^{2}}{\left|a_{1}\right|}\left(\beta_{2}\|g\|+1\right) .
$$

It is enough to put $\beta_{3}=\max \left\{\beta_{2},\left(N^{2} /\left|a_{1}\right|\right)\left(\beta_{2}\|g\|+1\right)\right\}$.

The next steps are analogous. If $k \in\{2,3, \ldots, 2 n\}$ we put $d_{k}=\left(1 / d_{k-1}\right)$ $\cdot \varphi\left(x_{k-1}\right)$ and thus we have

$$
\begin{aligned}
& \frac{\left|a_{1}\right|}{N^{k-1}} \leq\left|d_{k}\right| \leq N^{k-1}\left|a_{1}\right| \quad \text { if } k \text { is odd, } \\
& \frac{1}{N^{k-1}\left|a_{1}\right|} \leq\left|d_{k}\right| \leq \frac{N^{k-1}}{\left|a_{1}\right|} \quad \text { if } k \text { is even. }
\end{aligned}
$$

Also we define

$$
\beta_{k}= \begin{cases}\max \left\{\beta_{k-1}, \frac{N^{k-1}}{\left|a_{1}\right|}\left(\beta_{k-1}\|g\|+1\right)\right\} & \text { if } k \text { is odd, } \\ \max \left\{\beta_{k-1}, N^{k-1}\left|a_{1}\right|\left(\beta_{k-1}\|f\|+1\right)\right\} & \text { if } k \text { is even. }\end{cases}
$$

Proof of Theorem 5. Put $r=R / 2$ in Lemma 6. Thus $B(f, r) \subset B(F, R)$ and $B(g, r) \subset B(G, R)$. Define

$$
\varepsilon=\min \left\{\frac{\mu}{2}, \frac{r}{\max \left\{\beta_{1}, \ldots, \beta_{2 n}\right\}}\right\}
$$

where the numbers $\beta_{i}$ are chosen as in Lemma 8. It is enough to show that

$$
B(f g, \varepsilon) \subset B(f, r) \cdot B(g, r) .
$$

Indeed, let $\varphi \in B(f g, \varepsilon)$. Use Lemma 8 to pick functions $\zeta, \psi \in C$ with $\varphi=\zeta \psi$ and $\|f-\zeta\|_{i}<\beta_{i} \varepsilon,\|g-\psi\|_{i}<\beta_{i} \varepsilon$ for $i=1, \ldots, 2 n$. Hence

$$
\|f-\zeta\|<\varepsilon \max \left\{\beta_{1}, \ldots, \beta_{2 n}\right\} \leq r
$$

and analogously, $\|g-\psi\|<r$.

3. Some applications. Let us finish our paper with applications of Propositions 1, 2 and Theorem 5. Namely, we will show that if $\Phi \in\{+, \cdot, \min$, $\max$, then $\Phi^{-1}(E)$ is residual in $C \times C$ whenever $E$ is residual in $C$. Recall that a residual set is the complement of a set of the first category in a topological space. (See $[\mathrm{O}]$.)

Proposition 9. Let $X, Z$ be topological Baire spaces and let $E \subset X$ be a residual set. If $\Phi: Z \rightarrow X$ is a continuous mapping such that $\Phi(U)$ is of the second category for every nonempty open set $U \subset Z$, then $\Phi^{-1}(E)$ is a residual set.

Proof. Pick a dense $G_{\delta}$ set $F \subset E$. Let $F=\bigcap_{n=1}^{\infty} E_{n}$ where $E_{n}$ are

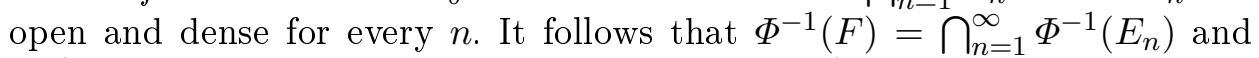
$\Phi^{-1}(F)$ is of type $G_{\delta}$. It suffices to show that $\Phi^{-1}(F)$ is dense. Let $U \subset Z$ 
be nonempty open. By the assumption, $\Phi(U) \cap F \neq \emptyset$. So pick $z \in U$ with $\Phi(z) \in F$. Hence $z \in U \cap \Phi^{-1}(F)$.

Corollary 10. Let $X$ be a complete metric space and let $\Phi: X \times X \rightarrow$ $X$ be a continuous mapping such that $\operatorname{Int}\left(\Phi\left(B_{1} \times B_{2}\right)\right) \neq \emptyset$ for any two balls $B_{1}, B_{2} \subset X$. Then $\Phi^{-1}(E)$ is residual for every residual set $E \subset X$.

Corollary 11. Let $\Phi: C \times C \rightarrow C$ where $\Phi \in\{+, \cdot, \min , \max \}$. Then $\Phi^{-1}(E)$ is residual for every residual set $E \subset C$.

If $E \subset C$ is residual, a property possessed by all continuous functions in $E$ is called typical. Several typical properties of continuous functions have been described in [Br]. In particular, the famous Banach-Mazurkiewicz theorem states that a typical function in $C$ is nondifferentiable at every point. Note that, for $E$ consisting of nowhere differentiable functions in $C$, the assertion of Corollary 11 was obtained in [Wa1] by a different argument.

The results of this paper, except for Theorem 3 and Corollary 4, are contained in [Wa2]. Here we provide a new shorter proof of Theorem 5.

Acknowledgements. We would like to thank V. V. Chistyakov, D. H. Fremlin, Sz. Głąb and J. Jachymski for their valuable comments.

\section{References}

[Br] A. Bruckner, Differentiation of Real Functions, 2nd ed., CRM Monograph Ser. 5, Amer. Math. Soc., Providence, RI, 1994.

[O] J. C. Oxtoby, Measure and Category, Springer, New York, 1971.

[Wa1] A. Wachowicz, Baire category and standard operations on pairs of continuous functions, Tatra Mt. Math. Publ. 24 (2002), 141-146.

[Wa2] —, On some residual sets, PhD dissertation, Łódź Technical Univ., Łódź, 2004 (in Polish).

Marek Balcerzak and Artur Wachowicz

Institute of Mathematics

Łódź Technical University

Wólczańska 215

93-005 Łódź, Poland

E-mail: mbalce@p.lodz.pl aw314@poczta.fm
Władysław Wilczyński Faculty of Mathematics University of Łódź Banacha 22

90-238 Łódź, Poland E-mail: wwil@uni.lodz.pl

Received November 30, 2004

Revised version March 21, 2005 